\title{
Management of persistent vulvo vaginal candidosis due to azole-resistant Candida glabrata
}

\author{
D J White, E M Johnson, D W Warnock
}

\begin{abstract}
Case report-Subjects-Three cases are described of long-standing vaginal candidosis due to Candida glabrata. These had failed to respond to local and systemic antifungals. In each case the infecting strain appeared resistant to a range of azole drugs in vitro.

Clinical course-Case one-This patient recovered following prolonged treatment with oral itraconazole in combination with oral and vaginal nystatin. Case two. Yeasts were eradicated from this patient following cyclical treatment with oral dydrogesterone; prolonged vaginal treatment with nystatin may have helped. Case three. This patient did not respond to a prolonged course of oral itraconazole in combination with vaginal and oral nystatin, oral medroxyprogesterone or intravaginal boric acid. Eradication of $C$ glabrata was finally achieved by local application of $1 \%$ gentian violet. Shortly after eradication of the $\boldsymbol{C}$ glabrata infection, both Case two and Case three developed infections with other Candida species responsive to azole antifungals.
\end{abstract}

(Genitourin Med 1993;69:112-114)

\section{Introduction}

Although Candida albicans is the usual cause of vulvovaginal candidosis, other members of the genus are sometimes involved. The second most common yeast recovered from the genital tract of women with vaginitis is Candida glabrata (formerly Torulopsis glabrata) which accounts for about $5 \%$ of vaginal infections. ${ }^{1}$ Infections with this organism are apt to be mild and it is not often associated with florid clinical signs.

Most women with vulvovaginal candidosis respond well to local or oral antifungal treatment. In some, however, the infection is recurrent, while others have persistent symptoms that fail to respond to treatment. Azole drug resistance has seldom been implicated as the cause of treatment failure in women with persistent vaginitis. However, recent reports indicate that even short-term treatment can lead to the rapid development of drug resistance in patients with $C$ glabrata infection. ${ }^{2}$ This paper describes three cases of persistent vaginal candidosis, unresponsive to azole drug treatment. In each case, laboratory testing of the $C$ glabrata isolates showed that the organisms were resistant to a range of azole antifungal drugs.

\section{Case histories}

All three women were otherwise well. A range of investigations including full blood count, random blood sugars, T-cell subsets and lymphocyte proliferations including those to whole $C$ albicans antigen were normal.

\section{Case 1}

A 38 year old woman presented with a 3 year history of persistent vaginal discharge, pruritus, external dysuria and marked superficial dyspareunia. Her symptoms worsened before menstruation. Numerous vaginal swabs had yielded heavy growths of yeast. She had been treated with nystatin and with topical and oral azole antifungals without response. Midstream urine specimens had yielded significant growths of coliforms, but her dysuria had not responded to the antibiotics prescribed. On examination, she was found to have a thick white vaginal discharge with minimal vulvovaginitis. Large numbers of yeasts were seen in a vaginal smear and a heavy growth of a Candida sp. was obtained in culture.

Treatment with fluconazole $(150 \mathrm{mg}$ once weekly) was commenced, but four weeks later her symptoms and signs were unchanged. Yeasts were seen on microscopy of a high vaginal swab which subsequently yielded a heavy growth of Candida glabrata. Oral fluconazole was discontinued and treatment with oral itraconazole commenced $(200 \mathrm{mg}$ twice daily for one day followed by $200 \mathrm{mg}$ twice daily three times weekly). Three weeks later her symptoms were much improved, although she still complained of marked vulval irritation. No evidence of vaginal yeasts was found on either microscopy or culture. Treatment with itraconazole $(200 \mathrm{mg}$ twice daily for one day per week) was continued for a further four weeks. In addition, oral nystatin (100000 units four times daily) and nystatin pessaries (500000 units nightly) were prescribed. Her vulval symptoms settled after four months. All antifungals were then discontinued.

\section{Case 2}

A 31 year old woman presented with a 2 year history of recurrent vaginal discharge, marked superficial dyspareunia and mild vulval irritation. Numerous vaginal swabs had yielded heavy growths of yeast. Her symptoms had not responded to a range of intravaginal imidazole antifungals, but a transient improve- 
ment had occurred following prolonged courses of nystatin pessaries and single doses of oral fluconazole $(150 \mathrm{mg})$. Her symptoms were worse after intercourse and before menstruation. On examination she was found to have a thick white vaginal discharge with minimal vulvovaginitis. Many yeasts were seen on microscopy and culture yielded a heavy growth of Candida glabrata.

Treatment with econazole pessaries (250 mg once weekly) was started, but 3 months later her symptoms were unchanged and many yeasts were again seen on microscopy with a heavy growth of $C$ glabrata in culture. Treatment was changed to nystatin pessaries (100 000 units at night). Three months later she reported that her symptoms had improved while using the pessaries, but had recurred when the pessaries were omitted for more than two or three days. Examination was normal and no yeasts were seen on microscopy or isolated in culture. However, she had been using nystatin pessaries for the previous few days. She also complained of long-standing symptoms, mostly of mood alteration, and pre-menstrual tension (PMT). Treatment with dydrogesterone $(10 \mathrm{mg}$ twice daily for days 14 to 28 of her cycle) was commenced. One month later she reported complete resolution of her symptoms of vaginitis but considerable worsening of her PMT. The dydrogesterone was therefore stopped. Vaginal examination was normal and fungal culture was negative.

A further episode of vulvovaginitis occurred 2 weeks after stopping the dydrogesterone, but this time the symptoms differed markedly with pronounced itching and minimal discharge. A high vaginal swab yielded a heavy growth of $C$ albicans and there was a prompt symptomatic response to fluconazole ( $100 \mathrm{mg}$ daily for 2 weeks). The patient failed to return for follow up for a further 3 months, but continued to have monthly episodes of vaginal candidosis characterised by marked itching and minimal discharge. Subsequently, $C$ albicans was again isolated. Her symptoms have since been controlled by the use of one econazole pessary $(250 \mathrm{mg})$ every two weeks.

\section{Case 3}

This 42 year old woman presented with a 5 year history of vaginal discharge and vulval itching and discomfort. Dyspareunia had almost completely precluded sexual intercourse. Previously, while she had been using a combined oral contraceptive, her symptoms had shown no variation with the menstrual cycle, but since this had been stopped 3 years previously, her symptoms had become markedly worse mid cycle when her labia minora became very swollen and oedematous. Numerous vaginal swabs had yielded heavy growths of yeast, but no clinical response had occurred despite more than 15 different courses of intravaginal imidazole antifungals and several courses of intravaginal nystatin. Nor had oral treatment with single doses of fluconazole $(150 \mathrm{mg})$ or itraconazole
(200 mg twice daily for one day) produced any symptomatic improvement. On examination, typical signs of vulvovaginal candidosis were found with marked vulvitis, vaginitis, and a profuse curdy discharge. Large numbers of yeasts were seen on microscopy and a heavy growth of $C$ glabrata was obtained from a high vaginal swab.

There was neither a symptomatic nor a mycological response to itraconazole $(200 \mathrm{mg}$ twice daily for 3 days followed by $100 \mathrm{mg}$ once daily) combined with nightly nystatin pessaries and oral nystatin (one million units 6 hourly for 2 weeks). Nor was there a response to medroxyprogesterone acetate (10 mg three times daily for 2 weeks). Intravaginal treatment with boric acid in gelatin capsules $(600 \mathrm{mg}$ ) was given twice daily for four weeks during which two cultures yielded a light growth of $C$ glabrata. Again there was no change in symptoms. She was then painted intravaginally with $1 \%$ gentian violet. One week later her symptoms were unchanged and yeast cells were seen on microscopy. However, culture of a high vaginal swab yielded Candida parapsilosis. In the meantime her treatment had been changed to flucytosine (500 mg orally four times daily), nightly nystatin pessaries and oral nystatin (1 million units six hourly). Two weeks later she returned with a photosensitive eruption. Flucytosine was stopped, but the oral and vaginal nystatin were continued. All subsequent vaginal cultures have been negative. Four months later her symptoms of itching were much improved, but the dyspareunia and vulval oedema were unchanged and a vulval biopsy showed only non-specific inflammation. She has since shown some improvement with intravaginal and vulval steroids.

\section{Antifungal sensitivity tests}

Antifungal drug sensitivity tests were carried out by preparing doubling dilutions of fluconazole, itraconazole, ketoconazole, flucytosine or nystatin in Sabourauds dextrose agar (glucose, $40 \mathrm{~g} / \mathrm{l}$; peptone, $20 \mathrm{~g} / \mathrm{l}$; agar, $30 \mathrm{~g} / \mathrm{l}$ ) or high-resolution medium in phosphate buffered agar (Pfizer Ltd, UK). The tests were performed with an inoculum concentration of $2 \times 10^{5}$ cells $/ \mathrm{ml}$ and the results were recorded after incubation at $37^{\circ} \mathrm{C}$ for $48 \mathrm{~h}$. The lowest drug concentration at which a strain showed no visible growth was considered to be the minimum inhibitory concentration (MIC).

The results (table) suggest that the $C$ glabrata isolates from cases 1 and 2 were resistant to fluconazole and itraconazole with an intermediate sensitivity to ketoconazole, whilst the isolate from case 3 was highly resistant to all three drugs. All isolates, however, were sensitive to nystatin. In contrast, the isolates of $C$ albicans and $C$ parapsilosis that had replaced the $C$ glabrata infections in cases 2 and 3 respectively were sensitive to all the drugs tested. 
Table MICs of fluconazole, itraconazole, ketoconazole, flucytosine and nystatin for yeast strains recovered from three cases of chronic vaginal candidosis.

\begin{tabular}{lllclll}
\hline Case & Organism & Fluconazole & Itraconazole & Ketoconazole & Flucytosine & Nystatin \\
\hline 1 & C glabrata & 64 & 32 & 4 & - & $<0.25$ \\
2 & C glabrata & $>64$ & 32 & 8 & - & $<0.25$ \\
& Calbicans & 0.5 & 0.25 & 8 & - & $<0.25$ \\
3 & C glabrata & $>64$ & $>64$ & 64 & $<0.25$ & $<0.25$ \\
& C parapsilosis & 1.0 & 0.25 & $>0.25$ & $<0.25$ & 0.5
\end{tabular}

${ }^{\star} 0.25 \mathrm{mg} / \mathrm{l}$ is equivalent to 1400 units of nystatin.

\section{Discussion}

Although azole antifungals are widely used, there have been few verified reports of resistant strains emerging during treatment of vaginal candidosis. By comparison with $C$ albicans strains, most strains of $C$ glabrata are intrinsically less sensitive to azole antifungals. Moreover because this organism is haploid (unlike $C$ albicans which is diploid) drug resistance is more likely to develop during treatment. ${ }^{3}$ It is therefore important to identify yeast isolates from patients presenting with vaginal candidosis that is clinically unresponsive to azole antifungals.

Although treatment failure can be due to other causes, antifungal drug resistance should be considered if yeasts other than $C$ albicans, and particularly $C$ glabrata, are isolated from patients with chronic vaginitis Although the result of MIC determinations with azole drugs are difficult to interpret, the MICs obtained for the $C$ glabrata strains recovered from our patients are high. In all three cases the patients had received numerous courses of azole antifungals. However, without pre-treatment isolates for comparison, it is impossible to tell whether these cases represent emergence of resistance during treatment.

Local nystatin was clearly ineffective in cases 1 and 3 , but it is possible in case 2 that long term local treatment with nystatin did prove helpful. The only culture taken before cyclical dydrogesterone was started was negative although a nystatin pessary had been used on the morning of the sample. Symptomatically, however, as with previous courses of nystatin, she had not improved and her vaginal symptoms only resolved completely after commencement of cyclical dydrogesterone. It seems probable therefore that this was a false negative culture due to a high local concentration of nystatin in the vagina at the time the swab was taken. It is difficult to explain why nystatin should fail to eradicate yeasts which appeared quite sensitive in vitro to this drug, but it seems that, in this clinical situation and with local intravaginal treatment, in vitro sensitivities to polyene antifungals may not reflect sensitivity in vivo.

Redondo et $\mathrm{al}^{4}$ have described similar cases of chronic vaginitis due to azoleresistant $C$ glabrata strains. Their patients included one unresponsive to all other treatment who finally responded to intravaginal boric acid. This was ineffective in the one patient on whom it was tried but $1 \%$ gentian violet painted intravaginally was ultimately effective.

Although depot medroxyprogesterone has been used to treat recurrent vulvovaginal candidosis, ${ }^{5}$ cyclical oral medroxyprogesterone or dydrogesterone are not recognised treatments. We have, however, seen resolution of recurrent candidosis in some women who, by chance, have been commenced on cyclical dydrogesterone for PMT (White, unpublished observations). Two weeks of oral medroxyprogesterone was ineffective in Case 1 but cyclical dydrogesterone was probably the effective treatment in Case 2. Cyclical oral progestagens may be worth investigating further as a treatment for chronic or recurrent vaginal candidosis.

In conclusion we suggest that cases of recurrent candidosis should be treated initially with high dose oral azoles in combination with oral and vaginal nystatin. If this fails then other therapies including vaginal boric acid and vaginal painting with $1 \%$ gentian violet should be considered. The role of progestagens is not clear, but may be worth considering, perhaps in combination with the initial treatment.

In two of our patients, symptoms of associated vulvitis were very slow to clear and eradication of the drug resistant $C$ glabrata strain was closely followed by recolonisation with another, drug-sensitive yeast which proved easier to treat. Treatment success should therefore be based on evaluation of the results of speciated vaginal cultures.

1 Oriel JD, Partridge BM, Denny MJ, Coleman JC. Genita yeast infections. $B M \mathcal{F}$ 1972;4:761-4.

2 Warnock DW, Burke J, Cope NJ, Johnson EM, Von Fraunhofer NA, Williams EW. Fluconazole resistanc in Candida glabrata. Lancet 1988;ii: 1310.

3 Kerridge D, Nicholas RO. Drug resistance in the opportunistic pathogens Candida albicans and Candida glabruta. $\mathcal{F}$ Antimicrob Chemother 1986;18 Suppl B:39-49.

4 Redondo LV, Lynch M, Schmitt C, Cook R, Sobel J. Torulopsis glabrata vaginitis: clinical aspects and suscepTorulopsis glabrata vaginitis. clinical aspects and suscep1990;76:651-5.

5 Dennerstein G. Depo-provera in the treatment of recurrent vulvo-vaginal candidiasis. $f$ Reprod $\mathrm{Ie}_{\mathrm{e}}$ 1986;31:801-3. 